\title{
Management of Differentiated Thyroid Cancer: The Standard of Care
}

\author{
Anca M. Avram ${ }^{1,2}$, Katherine Zukotynski ${ }^{3-5}$, Helen Ruth Nadel $^{6}$, and Luca Giovanella ${ }^{7,8}$ \\ ${ }^{1}$ Division of Nuclear Medicine, Department of Radiology, University of Michigan, Ann Arbor, Michigan; ${ }^{2}$ Division of Endocrinology, \\ Department of Internal Medicine, University of Michigan, Ann Arbor, Michigan; ${ }^{3}$ Departments of Medicine and Radiology, McMaster \\ University, Hamilton, Ontario, Canada; ${ }^{4}$ Department of Medical Imaging, Schulich School of Medicine and Dentistry, Western \\ University, London, Ontario, Canada; ${ }^{5}$ Department of Radiology, University of British Columbia, Vancouver, British Columbia, \\ Canada; ${ }^{6}$ Lucile Packard Children's Hospital at Stanford, Stanford University School of Medicine, Stanford, California; ${ }^{7}$ Clinic for \\ Nuclear Medicine and Competence Center for Thyroid Diseases, Imaging Institute of Southern Switzerland, Bellinzona, Switzerland; \\ and ${ }^{8}$ Clinic for Nuclear Medicine, University Hospital and University of Zurich, Zurich, Switzerland
}

In the past decade, the management of differentiated thyroid cancer (DTC) underwent a paradigm shift toward the use of risk stratification with the goal of maximizing the benefit and minimizing the morbidity of radioiodine $\left({ }^{131} I\right)$ therapy. ${ }^{131} I$ therapy is guided by information derived from surgical histopathology, molecular markers, postoperative diagnostic radioiodine scintigraphy, and thyroglobulin levels. ${ }^{131}$ I is used for diagnostic imaging and therapy of DTC based on physiologic sodium-iodine symporter expression in normal and neoplastic thyroid tissue. We summarize the essential information at the core of multidisciplinary DTC management, which emphasizes individualization of ${ }^{131} \mathrm{I}$ therapy according to the patient's risk for tumor recurrence.

Key Words: differentiated thyroid cancer; state of the art; guidelines; clinical management

J Nucl Med 2022; 63:189-195

DOI: 10.2967/jnumed.121.262402

N

andard-of-care management for differentiated thyroid cancer (DTC) is risk-adapted and typically includes surgery, ${ }^{131} \mathrm{I}$ therapy, and thyroid hormone therapy. In rare cases of radioiodinerefractory disease, symptomatic relief and slowing of metastatic disease progression may be provided by external radiotherapy, radiofrequency ablation, and multikinase or tyrosine kinase inhibitors.

\section{EPIDEMIOLOGY AND CLASSIFICATION}

Thyroid neoplasms are the most common endocrine tumors (annual incidence, 8-9 cases/100,000 people), and DTC accounts for more than $90 \%$ of cases. The rising DTC incidence in recent years may reflect a combination of increased imaging and a concomitant true rise in incidence (1). DTC is biologically and functionally heterogeneous, with different molecular pathways impacting cancer cell biology. In particular, BRAF V600E mutation is associated with reduced expression of all thyroid-specific genes involved in iodine

\footnotetext{
Received Apr. 4, 2021; revision accepted Aug. 5, 2021.

For correspondence or reprints, contact Anca M. Avram (ancaa@umich. edu).

Published online Aug. 19, 2021.

COPYRIGHT (C) 2022 by the Society of Nuclear Medicine and Molecular Imaging.
}

metabolism, resulting in variable decreased responsiveness to ${ }^{131}$ I therapy (2). The clinical, pathologic, and molecular characteristics of DTC are summarized in Supplemental Table 1 (supplemental materials are available at http://jnm.snmjournals.org) (3).

\section{DIAGNOSIS}

The most common clinical presentation of DTC is as an incidental thyroid nodule. Neck ultrasound, serum thyroid-stimulating hormone (TSH), and thyroid scintigraphy are used to select highrisk nodules for fine-needle aspiration. Sonomorphologic nodule features are used in the Thyroid Imaging Reporting and Data System, a standardized risk assessment for thyroid malignancy. In the absence of suggestive cervical lymph nodes, fine-needle aspiration is discouraged for nodules smaller than $1 \mathrm{~cm}$, and the decision to aspirate larger nodules is guided by the Thyroid Imaging Reporting and Data System score in the context of nodule size.

\section{SURGICAL TREATMENT}

Traditionally, total or near-total thyroidectomy was performed on most DTC patients, with lobectomy reserved for cytologically indeterminate nodules or patients with unifocal microcarcinoma (i.e., papillary thyroid carcinoma $\leq 1 \mathrm{~cm}$ ). Currently, lobectomy is suggested for patients with unifocal intrathyroidal low-risk DTC up to $4 \mathrm{~cm}$ in the absence of additional risk factors (i.e., no clinical evidence of nodal metastases, cN0) (4). The management of lowrisk DTC between 2 and $4 \mathrm{~cm}$ remains a topic of debate, and total thyroidectomy is still frequently advised (5). Active surveillance is an alternative to lobectomy for unifocal papillary thyroid microcarcinoma with no extracapsular extension or lymph node metastases (6). The decision for active surveillance is based primarily on age-related risk of progression, individual surgical risk factors, and patient preference (7).

Cervical lymph nodal metastases occur in $20 \%-60 \%$ of patients with DTC, and this nodal involvement varies from clinically relevant macrometastases to seemingly clinically irrelevant micrometastases $(8,9)$. When lymph nodal metastases are diagnosed preoperatively, central or lateral neck compartment dissection reduces the risk of locoregional recurrence. Prophylactic central neck dissection may improve regional control for invasive tumors (T3-T4) but is discouraged for low-risk DTC because potentially associated morbidities (i.e., hypoparathyroidism and recurrent 
laryngeal nerve damage) are not justified by a significant clinical benefit (10).

\section{POSTOPERATIVE MANAGEMENT}

Postoperative evaluation includes thyroglobulin measurement, neck ultrasound, and diagnostic radioiodine $\left({ }^{131} \mathrm{I}\right.$ or $\left.{ }^{123} \mathrm{I}\right)$ wholebody scanning (WBS), which is helpful to identify persistent disease and characterize tumor ${ }^{131} \mathrm{I}$ avidity. Tumor biology information encrypted in the molecular profile may also help determine indications for ${ }^{131} \mathrm{I}$ treatment and modulate treatment intensity; however, further studies are required before molecular profiling can be incorporated into clinical practice. Institutional management protocols are established by multidisciplinary teams based on the local availability and expertise of the surgical, pathology, radiology, and laboratory components integral to the DTC treatment algorithm (11).

\section{Postoperative ${ }^{131}$ I Therapy}

The goal of ${ }^{131}$ I therapy is determined on integration of clinical-pathologic, laboratory, and imaging information, using standardized definitions as follows (12).

Remnant Ablation. Remnant ablation is intended to eliminate postoperative normal thyroid tissue remnants in low-risk DTC in order to achieve an undetectable serum thyroglobulin level, facilitating follow-up and early detection of relapse. Thyroid remnant ablation also enables high-sensitivity posttherapy WBS for diagnosis and localization of any residual disease after surgery, such as unsuspected regional cervical metastases or distant metastatic disease.

Adjuvant Treatment. Adjuvant ${ }^{131} \mathrm{I}$ treatment in patients with suspected microscopic metastatic disease is based on histopathologic risk factors that predict tumor spread beyond the thyroid gland, with the intention of irradiating and eliminating occult infraradiologic residual disease in the neck or other occult micrometastases to improve recurrence-free survival.

Treatment of Known Disease. ${ }^{131} \mathrm{I}$ treatment in patients with known residual or metastatic disease has the goal of eliminating iodine-avid regional or distant metastases in order to achieve cure or remission, reduce persistent or recurrent disease, and improve overall prognosis.

\section{Preparation for ${ }^{131}$ I Therapy}

Evaluation with radioiodine scintigraphy and ${ }^{131} \mathrm{I}$ therapy is scheduled to take place at a minimum of $4 \mathrm{wk}$ after surgery, which allows time to prepare the patient and reach the necessary postoperative thyroglobulin plateau levels, used as a marker for residual thyroid tissue or metastatic thyroid cancer after total thyroidectomy. Thyroglobulin levels must always be interpreted in the context of concomitant TSH level (unstimulated vs. stimulated thyroglobulin) and type of TSH stimulation (endogenous vs. exogenous) (13). Patient preparation for optimal ${ }^{131}$ I uptake by residual thyroid tissue and metastatic disease includes 1-2 wk of a low-iodine diet (Supplemental Table 2) and adequate TSH stimulation (TSH $\geq 30$ $\mathrm{mIU} / \mathrm{L}$, measured $1-3 \mathrm{~d}$ before ${ }^{131} \mathrm{I}$ administration) by either thyroid hormone withdrawal (THW) or recombinant human TSH (rhTSH) stimulation (14). For female patients of childbearing age (12-50 y), a negative pregnancy test is required within $72 \mathrm{~h}$ of ${ }^{131} \mathrm{I}$ administration or before the first rhTSH injection (if used), unless the patient has undergone hysterectomy or is postmenopausal.

There are 2 main approaches to TSH stimulation, which is necessary for increasing Na-I symporter expression and function in metastatic lesions (and residual thyroid tissue), with the goal of increasing the diagnostic sensitivity of ${ }^{131} \mathrm{I}$ scintigraphy and absorbed radiation dose to target lesions.

Endogenous TSH Stimulation. Endogenous TSH stimulation is through thyroid hormone deprivation after total thyroidectomy, thus inducing a hypothyroid state: the hypothyroid stimulation protocol (THW) has 2 variants: levothyroxine withdrawal for $4 \mathrm{wk}$ or levothyroxine/liothyronine substitution for the first $2 \mathrm{wk}$.

Exogenous TSH Stimulation. For exogenous TSH stimulation, the patient continues levothyroxine treatment, and TSH is elevated through administration of rhTSH (thyrotropin alfa [Thyrogen; Genzyme] stimulation protocol): $0.9 \mathrm{mg}$ of rhTSH administered intramuscularly on 2 consecutive days, followed by ${ }^{131} \mathrm{I}$ therapy administered at 48-72 h.

The choice of preparation method (THW vs. rhTSH) needs to be individualized for each patient. There is general agreement that for normal thyroid tissue (i.e., thyroid remnant), rhTSH and THW stimulation are equivalent, because normal thyroid tissue has constitutive high expression of highly functional Na-I symporter and does not require prolonged TSH stimulation for adequate ${ }^{131} \mathrm{I}$ uptake and retention. However, metastatic thyroid cancer is less dense and has poorer functionality of the Na-I symporter; therefore, TSH elevation over time (area under the curve of TSH stimulation) is important to promote increased ${ }^{131} \mathrm{I}$ uptake and retention in tumors $(15,16)$. In the setting of distant metastases, THW preparation and dosimetry-guided ${ }^{131}$ I therapy are favored, when clinically safe and feasible (17-19).

\section{${ }^{131}$ I THERAPY}

There are 2 approaches to delivering ${ }^{131}$ I therapy: the theranostic approach, which integrates the information obtained with postoperative diagnostic radioiodine $\left({ }^{123} \mathrm{I}\right.$ or $\left.{ }^{131} \mathrm{I}\right)$ scans in the management algorithm, and the risk-based approach based on clinical-pathologic factors and institutional protocols. Which of these 2 approaches is chosen depends on local factors, including the quality of surgery; the availability of, and expertise with, various imaging modalities; and physician and patient preferences. Each approach has strengths and limitations, and no conclusive evidence regarding primary outcome measures is available for recommending one strategy over the other.

\section{Management of the Integration of Functional Diagnostic Radioiodine Imaging}

This theranostic approach to ${ }^{131} \mathrm{I}$ administration involves the acquisition of a postoperative diagnostic radioiodine $\left({ }^{123} \mathrm{I},{ }^{131} \mathrm{I}\right.$, or ${ }^{124}$ I) scan for planning ${ }^{131}$ I therapy. Diagnostic WBS is performed to identify and localize regional and distant metastatic disease and to determine the capacity of metastatic deposits to concentrate ${ }^{131} \mathrm{I}$. Depending on institutional protocols, the findings on diagnostic WBS may alter management, such as providing guidance for additional surgery; altering the prescribed ${ }^{131} \mathrm{I}$ therapy, either by adjusting conventional ${ }^{131}$ I activity or by performing dosimetry calculations to determine the maximum tolerated therapeutic ${ }^{131} \mathrm{I}$ activity for treatment of distant metastases; and avoiding unnecessary ${ }^{131}$ I therapy when diagnostic WBS finds no evidence of residual thyroid tissue or metastatic disease and the stimulated thyroglobulin is less than $1 \mathrm{ng} / \mathrm{mL}$ in the absence of interfering antithyroglobulin antibodies (the thyroglobulin cutoff should be adapted locally depending on the stimulation protocol and thyroglobulin assay) (17). Information acquired from the diagnostic WBS may also lead to additional functional metabolic imaging 
with ${ }^{18}$ F-FDG PET/CT when non-iodine-avid metastatic disease is suspected (on the basis of thyroglobulin elevation out of proportion to the findings on the diagnostic WBS). It is preferable for postoperative diagnostic scanning to be performed using integrated multimodality imaging (i.e., SPECT/CT), whenever available. SPECT/CT is relevant for assessing focal radioiodine uptake in the neck, for differentiating thyroid remnant from nodal metastasis, and for detecting metastases in normal-sized cervical lymph nodes (not appreciated on postoperative neck ultrasound). Scintigraphic evaluation with diagnostic WBS can identify pulmonary micrometastases (which are too small to be detected on routine chest radiographs and may remain undetected on CT; Fig. 1) and bone metastases at an early stage (i.e., before cortical disruption is visible on bone radiographs or CT; Fig. 2). Most importantly, since ${ }^{131}$ I therapy is most effective for smaller metastatic deposits, early identification of regional and distant metastases is important for successful therapy $(20,21)$. In a group of 320 thyroid cancer patients referred for postoperative ${ }^{131} \mathrm{I}$ therapy, diagnostic WBS with SPECT/CT detected regional metastases in 35\% of patients and distant metastases in $8 \%$ of patients. This scintigraphy information changed staging in $4 \%$ of younger, and $25 \%$ of older, patients (22). Clinical management was changed in $29 \%$ of patients when information from diagnostic WBS and stimulated thyroglobulin was integrated into the decision algorithm, as compared with a management strategy based on clinical and histopathology information alone (23). In 350 patients at intermediate and high risk of recurrence, a single ${ }^{131} \mathrm{I}$ therapeutic administration guided by postoperative diagnostic WBS information resulted in a complete response in $88 \%$ of patients with locoregional disease and $42 \%$ of patients with distant metastases (median follow-up of 3 y) (24). The information obtained with the diagnostic WBS reasonably predicts ${ }^{131} \mathrm{I}$ therapeutic localization and can be used for ${ }^{131}$ I therapy planning in the paradigm of thyroid cancer radiotheranostics $(22,25,26)$.

${ }^{124} \mathrm{I}$ is a positron emitter isotope with a 4.2-d half-life that has imaging characteristics superior to those of ${ }^{123} \mathrm{I}$ and ${ }^{131} \mathrm{I}$ scintigraphy. ${ }^{124} \mathrm{I}$ is expensive, with limited accessibility, and is not widely available; however, on the basis of its PET imaging capability, ${ }^{124}$ I is the ideal agent for pretherapy tumor and organ dosimetry calculations (27). A standardized protocol for ${ }^{124} \mathrm{I}$ PET/ CT acquisition, analysis, and quantification remains to be established.

\section{Risk-Adapted ${ }^{131}$ I Therapy Followed by ${ }^{131}$ I Scans with Diagnostic Intent}

The conventional approach, in which the nuclear medicine physician chooses an ${ }^{131}$ I activity based on local protocols, availability, experience with various imaging modalities, and patientrelated parameters, is widely used for thyroid remnant ablation, adjuvant treatment, and curative therapy of known structural disease. With this therapeutic approach, the prescribed ${ }^{131}$ I activity depends on the goal of ${ }^{131}$ I therapy as determined by the estimated risk for persistent or recurrent disease. Thyroid remnant ablation in low-risk patients is typically performed with a low ${ }^{131} \mathrm{I}$ activity (i.e., $1.1-1.85 \mathrm{GBq}[30-50$ $\mathrm{mCi}]$ ). Adjuvant ${ }^{131} \mathrm{I}$ therapy is performed with a slightly higher activity (i.e., 1.85-3.7 GBq [50-100 $\mathrm{mCi}]$, with some institutions extending this range to 5.55 $\mathrm{GBq}$ [150 mCi]). Treatment of known disease is performed by administering a high activity (i.e., 3.7-5.56 GBq [100-150 mCi] for advanced locoregional disease and 5.6 $-7.4 \mathrm{GBq}$ [150-200 $\mathrm{mCi}]$ for distant metastatic disease) (28). However, when diagnostic scintigraphy demonstrates diffuse homogeneous uptake throughout the lungs, simplified whole-body dosimetry should be performed to adjust the prescribed ${ }^{131}$ I activity so that pulmonary ${ }^{131} \mathrm{I}$ retention does not exceed $3 \mathrm{GBq}(80 \mathrm{mCi})$ after $48 \mathrm{~h}$, with the goal of minimizing the risk of radiationinduced lung toxicity (29). Administration of therapeutic ${ }^{131}$ I activities of at least $7.4 \mathrm{GBq}$ (200 mCi) for treatment of diffuse distant metastatic disease requires full whole-body or blood dosimetry calculations (24). Table 1 shows a suggested ${ }^{131}$ I therapy framework in DTC.

Posttherapy WBS is performed at $2-7 \mathrm{~d}$ after ${ }^{131} \mathrm{I}$ administration to localize the therapeutic ${ }^{131} \mathrm{I}$ and assess for regional and distant
FIGURE 1. Radioiodine theranostics for 63-y-old man with 2.2-cm regionally advanced papillary thyroid carcinoma and of 11 lymph nodes dissected, all 11 were positive for metastasis, in surgical specimen of total thyroidectomy. (A) Diagnostic $37-\mathrm{MBq}(1 \mathrm{mCi}){ }^{131} \mathrm{I}$ WBS, anterior projection, depicts multifocal neck activity and diffuse lung activity. ( $B$ and $C$, different anatomic levels within the neck, depicting lymph nodal metastases located in different cervical compartments) Neck SPECT/CT demonstrates iodine-avid soft-tissue nodules consistent with cervical nodal metastases. (D) Chest SPECT/CT demonstrates diffuse lung activity and branching pulmonary vasculature without definite lung nodules identified. Patient received dosimetry-guided 12.6-GBq (340 mCi) ${ }^{131}$ I treatment. (E) On posttherapy WBS at $3 \mathrm{~d}$, anterior projection demonstrates therapeutic ${ }^{131}$ I localization to cervical lymph nodal metastases and diffuse miliary pulmonary metastatic disease. 


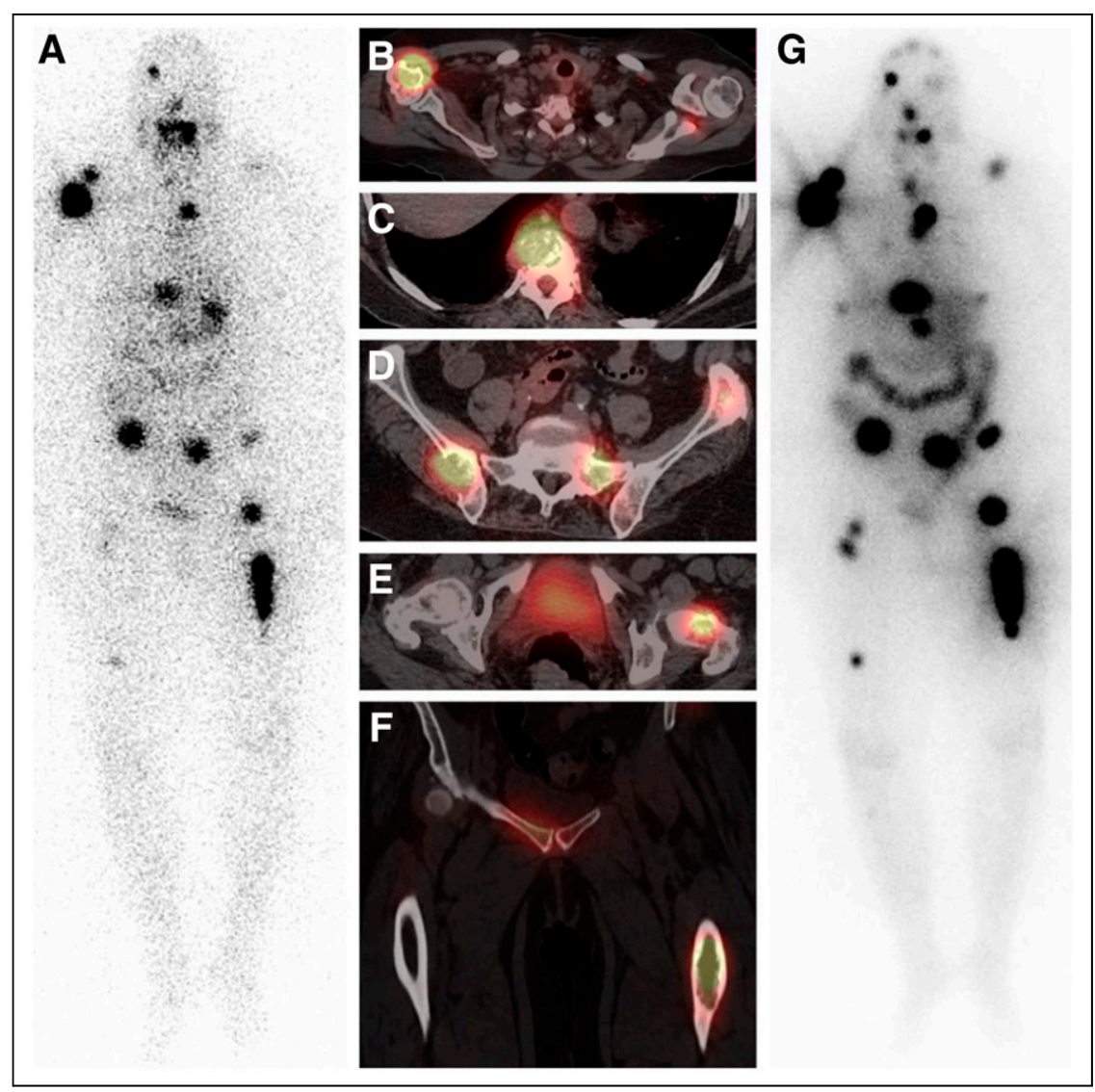

FIGURE 2. Radioiodine theranostics for $66-y$-old woman with $2.5-\mathrm{cm}$ widely invasive follicular thyroid carcinoma with osseous metastatic disease. (A-F) Diagnostic $37-\mathrm{MBq}(1 \mathrm{mCi}){ }^{131} \mathrm{I}$ WBS, anterior projection (A), depicts multifocal skeletal activity, which is further characterized on SPECT/CT as iodine-avid lytic osseous metastases involving right humerus $(B)$, vertebrae $(C)$, pelvis (D) left femoral neck $(E)$, and left femoral diaphysis $(F)$. Patient received dosimetry-guided $12-\mathrm{GBq}(325 \mathrm{mCi}){ }^{131}$ I treatment. (G) Posttherapy WBS, anterior projection, obtained at $2 \mathrm{~d}$ demonstrates therapeutic ${ }^{131} \mathrm{I}$ targeting of extensive iodine-avid multifocal osseous metastatic disease involving axial and proximal appendicular skeleton, with increased lesion conspicuity and numerous new foci detected as compared with diagnostic scan.

metastases. Campenni et al. reported in a cohort of 570 DTC patients at low risk or low-to-intermediate risk (pT1-pT3) that posttherapy WBS with SPECT/CT demonstrated metastases in 82 (14.4\%), of whom $73(90.2 \%)$ had a postsurgical nonstimulated thyroglobulin level of no more than $1 \mathrm{ng} / \mathrm{mL}$ (30). Furthermore, in 44 patients $(54 \%)$, stimulated thyroglobulin remained at no more than $1 \mathrm{ng} / \mathrm{mL}$, despite the presence of metastases on posttreatment scans (30). Therefore, postsurgical nonstimulated thyroglobulin levels cannot be used independently in deciding whether to pursue therapeutic ${ }^{131} \mathrm{I}$ administration, mainly in patients assigned as low risk on the basis of surgical pathology alone.

\section{THYROID HORMONE REPLACEMENT THERAPY}

After thyroidectomy, DTC patients require thyroid hormone (levothyroxine) replacement. The TSH target for levothyroxine therapy is based on a dynamic risk classification, thyroglobulin level, thyroglobulin trend over time, antithyroglobulin antibodies, and potential adverse effects of TSH suppression. For patients with a structurally incomplete response, the serum TSH is suppressed to less than $0.1 \mathrm{mU} / \mathrm{L}$. In the excellent treatment response category, the serum TSH is maintained at $0.5-2 \mathrm{mUI} / \mathrm{L}$ for intermediate-risk patients and at $0.1-0.5 \mathrm{mUI} / \mathrm{L}$ for high-risk patients. For patients with biochemically indeterminate or incomplete responses, the recommended serum TSH target is $0.1-0.5 \mathrm{mU} / \mathrm{L}(6)$.

\section{DTC MANAGEMENT IN CHILDREN}

DTC represents $1.8 \%$ of all cases of cancer in individuals less than $20 \mathrm{y}$ old. The incidence of pediatric thyroid cancer has increased, in part because of better techniques for diagnosis. A $4.43 \%$ increase in all stages of primarily papillary histology in 10- to 19-y-old non-Hispanic white patients, non-Hispanic black patients, and Hispanic patients was reported in a study that included 39 U.S. cancer registries (31).

Although $50 \%$ of adults older than $60 \mathrm{y}$ have thyroid nodules, only $5 \%-7 \%$ of children and young adults are diagnosed with thyroid nodules. However, thyroid cancer is diagnosed in $25 \%$ of thyroid nodules in the pediatric population (as compared with $10 \%-15 \%$ of thyroid nodules in adults). Assessment using the Thyroid Imaging Reporting and Data System is not applied in pediatric patients, as one study of 314 patients younger than $18 \mathrm{y}$ showed that $22 \%$ of cancers would be missed (32). Children with thyroid cancer have an increased incidence of regional and distant metastatic disease at presentation, as compared with adults, with cervical lymph nodal metastases diagnosed in $50 \%$ of cases and lung metastases in $20 \%$.

The recommended treatment for pediatric thyroid cancer is total thyroidectomy because of the higher incidence of bilateral and multifocal disease (30\% and $65 \%$, respectively) than in adults, as well as the greater likelihood for regional and distant metastasis at presentation (33). Central node dissection is recommended in the presence of locoregional cervical disease diagnosed by imaging and confirmed on fine-needle aspiration or identified during surgery. Although some have approached disease in a single lobe with lobectomy or a phased approach, a recent study by Zong et al. suggested that because of large lesions often involving both thyroid lobes in the pediatric age group, there is increased morbidity in staged resection (34). Lobectomy may be indicated in cases of follicular thyroid carcinoma that have certain characteristics, including a size of less than $4 \mathrm{~cm}$ and invasion of fewer than 3 vessels (minimally invasive follicular thyroid carcinoma).

The American Thyroid Association pediatric guidelines identify 3 risk categories dependent on risk of persistent disease: low risk, or disease confined to the thyroid with few central nodes and no macroscopic metastases; medium risk, or significant central and minimal lateral node involvement; and high risk, or either distant metastases or locally invasive tumor with extensive involvement of lateral neck lymph nodes (33).

Postoperatively, the use of diagnostic WBS and 24-h neck uptake at 6-12 wk after surgery and thyroglobulin testing is 
TABLE 1

Suggested Framework for ${ }^{131}$ I Therapy for DTC (28)

\begin{tabular}{lll}
\hline Strategy & \multicolumn{1}{c}{ Prescribed ${ }^{131}$ I activity } & Clinical context \\
\hline Risk-adapted ${ }^{131}$ I therapy & $1.11-1.85 \mathrm{GBq}(30-50 \mathrm{mCi})$ & Remnant ablation \\
& $1.85-3.7 \mathrm{GBq}(50-100 \mathrm{mCi})$ & Adjuvant treatment \\
& $3.7-5.6 \mathrm{GBq}(100-150 \mathrm{mCi})$ & $\begin{array}{c}\text { Treatment of small-volume locoregional } \\
\text { disease }\end{array}$ \\
Whole-body/blood dosimetry & $5.6-7.4 \mathrm{GBq}(150-200 \mathrm{mCi})$ & $\begin{array}{c}\text { Treatment of advanced locoregional } \\
\text { disease or small-volume distant } \\
\text { metastatic disease }\end{array}$ \\
& $\begin{array}{c}\geq 7.4 \mathrm{GBq}(\geq 200 \mathrm{mCi}), \text { maximum tolerable } \\
\text { safe activity }\end{array}$ & $\begin{array}{c}\text { Treatment of diffuse distant metastatic } \\
\text { disease }\end{array}$
\end{tabular}

All pediatric therapeutic ${ }^{131} \mathrm{I}$ activities are adjusted as multiplier based on $70-\mathrm{kg}$ adult body weight.

recommended. Although the American Thyroid Association guidelines suggest this for intermediate- and high-risk groups, many pediatric centers extend it to all risk groups. Apart from the fact that low-risk disease is less common in children, children have a higher risk of recurrence of DTC (35). Diagnostic WBS is now routinely performed with SPECT/CT (unenhanced CT) to evaluate for cervical, upper mediastinal, and pulmonary metastatic disease. Depending on the diagnostic WBS findings, the child may be referred back to surgery for resection of unsuspected bulky residual nodal metastases in unexplored neck compartments or may proceed with ${ }^{131} \mathrm{I}$ therapy for remnant ablation or treatment of locoregional or pulmonary metastatic disease, if present.

There is controversy as to how the ${ }^{131} \mathrm{I}$ treatment activity is determined. Some suggest an empiric dosing for initial ${ }^{131}$ I therapy in pediatrics, with dosimetry possibly relegated if follow-up ${ }^{131}$ I therapy is needed. A weight-based dose calculated by multiplying a $70-\mathrm{kg}$ adult by a $1-1.5 \mathrm{mCi} / \mathrm{kg}(37-56 \mathrm{MBq} / \mathrm{kg})$ activity has been used (36). American Thyroid Association guidelines do not specify recommended therapeutic ${ }^{131}$ I activities. Parisi et al. presented this algorithm: low-risk disease, 1.1-1.85 GBq (30-50 $\mathrm{mCi}$ ); higher-risk locoregional disease, 5.6-6.5 GBq (150-175 $\mathrm{mCi})$; and known or suspected pulmonary metastatic disease, 6.5-7.4 GBq (175-200 mCi). All these therapeutic ${ }^{131} \mathrm{I}$ activities are adjusted for body weight based on a $70-\mathrm{kg}$ adult weight (Table 1) (35). As with adults, routine posttherapy WBS is performed at 5-7 d after therapy.

The overall prognosis for pediatric DTC is good, with greater than a $98 \% 10$-y survival rate (37). In the small subset of children with refractory disease or disease that is not responsive to ${ }^{131} \mathrm{I}$ treatment, newer molecular therapies that target the known genetic alterations and molecular mutations may be used (38).

\section{RESPONSE ASSESSMENT AFTER PRIMARY TREATMENT AND FOLLOW-UP}

The combination of thyroglobulin measurement, neck ultrasound, and follow-up diagnostic WBS performed at 1-2 y after primary therapy is used to restratify the risk of recurrence according to the patient's response to initial therapy. This process of risk reassignment is called dynamic risk stratification and is predictive of long-term clinical outcome. The criteria for treatment response evaluation are summarized in Table 2 (4).
In patients with an excellent (complete) response, the risk of disease recurrence is $1 \%-4 \%$, which for intermediate-risk patients (whose initial recurrence risk is $36 \%-43 \%$ ) and for high-risk patients (whose initial recurrence risk is 68\%-70\%) represents a major change in risk. The clinical outcome in patients with a biochemically incomplete response is usually good: approximately $60 \%$ of patients have no evidence of disease over long-term follow-up; $20 \%$ of patients have persistently abnormal thyroglobulin without a structural correlate, and $20 \%$ of patients develop structurally identifiable disease over 5-10 y of follow-up. Patients with a biochemically indeterminate response generally do well: in $80 \%-90 \%$ of patients, nonspecific biochemical findings either remain stable or resolve over time with levothyroxine suppression therapy alone; however, up to $20 \%$ of these patients will eventually develop functional or structural evidence of disease progression and require additional therapy. Patients with a structurally incomplete response require multidisciplinary management tailored to their disease status (e.g., regional vs. distant metastases and iodine-avid vs. non-iodine-avid disease) (4); depending on the results of additional treatment, patients are restratified according to the criteria above.

\section{TREATMENT OF ADVANCED DISEASE}

Distant metastases develop in about $10 \%$ of DTC patients, commonly in the lungs, bone, brain, liver, and skin, and are the main cause of death (i.e., overall mortality of $65 \%$ at $5 \mathrm{y}$ and $75 \%$ at 10 years) (39).

The prognosis of metastatic DTC is variable, with 2 distinct phenotypes identified: indolent and aggressive (40). Patients with iodine-avid metastatic DTC tend to have a better prognosis, with 10 -y survival greater than $90 \%$, whereas non-iodine-avid metastatic DTC has a dire 10-y survival of 10\% (41). Younger patients and those with single-organ metastases and a low disease burden have the best outcome. The mainstay of treatment is TSH suppression and ${ }^{131}$ I therapy as long as the disease remains radioiodineavid. About two thirds of patients have radioiodine-avid distant metastases, and one third of them will achieve remission after multiple radioiodine treatments (20). Approximately $15 \%-20 \%$ of patients with metastatic DTC and most patients with Hürthle cell thyroid cancer are refractory to radioiodine, and overall survival for these patients ranges between 2.5 and $4.5 \mathrm{y}(20,42)$. 


\begin{tabular}{lc}
\hline Response & Criteria \\
\hline Excellent (complete) & $\begin{array}{c}\text { No clinical, biochemical, or structural evidence of disease; definition: negative imaging and } \\
\text { either suppressed thyroglobulin }<0.2 \mathrm{ng} / \mathrm{mL} \text { or stimulated thyroglobulin }<1 \mathrm{ng} / \mathrm{mL}\end{array}$ \\
\hline Biochemically incomplete & $\begin{array}{c}\text { Abnormal thyroglobulin (suppressed thyroglobulin }>1 \mathrm{ng} / \mathrm{mL} \text { or stimulated thyroglobulin } \\
>10 \mathrm{ng} / \mathrm{mL} \text { or rising anti-thyroglobulin antibody levels in absence of localizable disease } \\
\text { [i.e., negative imaging]) }\end{array}$ \\
\hline Structurally incomplete & $\begin{array}{c}\text { Persistent or new locoregional or distant metastases (any thyroglobulin) } \\
\text { Indeterminate }\end{array}$ \\
& $\begin{array}{c}\text { thyroglobulin }=1-10 \mathrm{ng} / \mathrm{mL} \text { or stable/declining anti-thyroglobulin antibody levels) or } \\
\text { structural findings that cannot be confidently classified as benign or malignant }\end{array}$ \\
\hline
\end{tabular}

Determining when a patient no longer responds to ${ }^{131} \mathrm{I}$ can be challenging. Factors impacting the specific clinical situation such as age, tumor histology, stage, residual radioiodine avidity, and ${ }^{18}$ F-FDG avidity should be evaluated (43). ${ }^{18}$ F-FDG PET/CT is particularly useful for identifying and localizing non-iodine-avid metastases and is used to evaluate patients with elevated thyroglobulin along with negative results on diagnostic WBS (44). In this setting, if lack of ${ }^{131} \mathrm{I}$ uptake has already been established on diagnostic WBS, a positive ${ }^{18} \mathrm{~F}$-FDG PET/CT result strongly supports the suspicion of ${ }^{131}$ I-negative or ${ }^{131}$ I-refractory disease, leading to changes in management by identifying patients who are unlikely to benefit from additional ${ }^{131}$ I therapy and instead qualify for alternative therapy (45). In addition, ${ }^{18} \mathrm{~F}-\mathrm{FDG}$ PET/CT has shown prognostic value in metastatic DTC, predicting the course of disease as aggressive or indolent (46). In radioiodine-refractory metastatic DTC, there is a survival disadvantage for patients with positive PET results as compared with those with negative PET results (42).

Ablative treatment for locoregional disease control (i.e., resection, vertebroplasty, external-beam radiation therapy, and thermal ablation) can provide symptomatic relief and delay the initiation of systemic therapy, whereas bisphosphonate or denosumab can delay the time to skeleton-related events (47). In cases of confirmed radioiodine-resistant metastatic disease progression, treatment with multikinase inhibitors (e.g., lenvatinib, sorafenib, vandetanib, cabozantinib, vemurafenib, dabrafenib/trametinib, and others) may induce periods of progression-free survival (rarely remission) without evidence of increased cancer-specific survival, and these drugs may be associated with side effects, such as hypertension, diarrhea, hand or foot skin reactions, rash, fatigue, mucositis, loss of appetite, and weight loss (48). The optimal time to start therapy, especially in asymptomatic patients, is unclear, nor is it clear which patients are likely to benefit in terms of increased quality-adjusted life years. As a rule, the decision to start molecular targeted therapies is based on a multidisciplinary team discussion. Such therapies are more likely to be used in patients who have negative findings on diagnostic WBS and symptoms that are not amenable to local therapy or who have progression of measurable lesions as defined by RECIST over the previous $12 \mathrm{mo}$, taking into consideration the tumor burden and the risk of local complications (49). The biologic mechanisms implicated in radioiodine refractoriness involve gain-of-function mutations in the MAPK signaling pathway, resulting in reduced Na-I symporter and other iodine-metabolizing gene expression. Experimental data showed that MAPK signaling pathway inhibition using MEK or
BRAF inhibitors restored radioiodine avidity. Subsequent clinical studies demonstrated that mutation-guided treatment using selective MEK inhibitors (selumetinib, trametinib), BRAF inhibitors (dabrafenib, vemurafenib), or a combination of BRAF inhibitor and MEK inhibitor is feasible and promising for redifferentiating radioiodine-refractory DTC, thereby permitting reapplication of ${ }^{131}$ I therapy. Preliminary data obtained on a small clinical series of 13 patients demonstrated restoration of ${ }^{131} \mathrm{I}$ avidity in $62 \%$ of patients who subsequently received ${ }^{131} \mathrm{I}$ treatment (median activity, 7.6 GBq [204.4 mCi]; range, 5.5-9.4 GBq [150-253 mCi]), resulting in durable disease control (median duration $>1 \mathrm{y}$ ) while not receiving chronic, expensive multikinase inhibitor therapy (50). ${ }^{131}$ I therapy remains the only known cure for metastatic radioiodine-sensitive DTC, and the use of a redifferentiating strategy to permit additional ${ }^{131} \mathrm{I}$ treatment for patients with radioiodine-refractory metastatic disease represents a promising therapeutic approach while minimizing exposure to kinase inhibitor therapy.

\section{CONCLUSION}

DTC is the most common endocrine malignancy. Although standard management including surgery and radioiodine therapy is successful in most cases, therapy should be tailored according to a risk stratification integrating the information from histopathology, molecular markers, postoperative thyroglobulin levels, and imaging studies. Ultimately, local multidisciplinary teams consider the availability of surgical, pathologic, nuclear medicine, and laboratory expertise and take into account individual patient preferences to guide appropriate therapy.

\section{DISCLOSURE}

No potential conflict of interest relevant to this article was reported.

\section{ACKNOWLEDGMENT}

We thank Vanessa Allen for preparing composite figures for this article.

\section{REFERENCES}

1. Davies L, Morris LG, Haymart M, et al. American Association of Clinical Endocrinologists and American College of Endocrinology disease state clinical review: the increasing incidence of thyroid cancer. Endocr Pract. 2015;21:686-696. 
2. Gulec SA, Ahuja S, Avram A, et al. A joint statement from the American Thyroid Association, the European Association of Nuclear Medicine, the European Thyroid Association, the Society of Nuclear Medicine and Molecular Imaging on current diagnostic and theranostic approaches in the management of thyroid cancer. Thyroid. 2021;31:1009-1019.

3. Rossi ED, Fadda G. Pathology and immunohistochemistry in thyroid tumors. In: Atlas of Thyroid and Neuroendocrine Tumor Markers. Giovanella L, ed. Springer; 2018:3-12.

4. Haugen BR, Alexander EK, Bible KC, et al. 2015 American Thyroid Association management guidelines for adult patients with thyroid nodules and differentiated thyroid cancer: the American Thyroid Association Guidelines task force on thyroid nodules and differentiated thyroid cancer. Thyroid. 2016;26:1-133.

5. Bilimoria KY, Bentrem DJ, Ko CY, et al. Extent of surgery affects survival for papillary thyroid cancer. Ann Surg. 2007;246:375-381.

6. Ito Y, Miyauchi A, Oda H. Low-risk papillary microcarcinoma of the thyroid: a review of active surveillance trials. Eur J Surg Oncol. 2018;44:307-315.

7. Davies L, Roman BR, Fukushima M, Ito Y, Miyauchi A. Patient experience of thyroid cancer active surveillance in Japan. JAMA Otolaryngol Head Neck Surg. 2019; 145:363-370.

8. Miller JE, Al-Attar NC, Brown OH, et al. Location and causation of residual lymph node metastasis after surgical treatment of regionally advanced differentiated thyroid cancer. Thyroid. 2018;28:593-600.

9. Roh JL, Park JY, Park CI. Total thyroidectomy plus neck dissection in differentiated papillary thyroid carcinoma patients: pattern of nodal metastasis, morbidity, recurrence, and postoperative levels of serum parathyroid hormone. Ann Surg. 2007;245:604-610.

10. Barczyński M, Konturek A, Stopa M, Nowak W. Prophylactic central neck dissection for papillary thyroid cancer. Br J Surg. 2013;100:410-418.

11. Tuttle RM, Ahuja S, Avram AM, et al. Controversies, consensus, and collaboration in the use of ${ }^{131} \mathrm{I}$ therapy in differentiated thyroid cancer: a joint statement from the American Thyroid Association, the European Association of Nuclear Medicine, the Society of Nuclear Medicine and Molecular Imaging, and the European Thyroid Association. Thyroid. 2019;29:461-470.

12. Van Nostrand D. The benefits and risks of I-131 therapy in patients with welldifferentiated thyroid cancer. Thyroid. 2009;19:1381-1391.

13. Mazzaferri EL, Kloos RT. Clinical review 128: current approaches to primary therapy for papillary and follicular thyroid cancer. J Clin Endocrinol Metab. 2001;86: 1447-1463.

14. Xiao J, Yun C, Cao J, et al. A pre-ablative thyroid-stimulating hormone with 30-70 $\mathrm{mIU} / \mathrm{L}$ achieves better response to initial radioiodine remnant ablation in differentiated thyroid carcinoma patients. Sci Rep. 2021;11:1348.

15. Pötzi C, Moameni A, Karanikas G, et al. Comparison of iodine uptake in tumour and nontumour tissue under thyroid hormone deprivation and with recombinant human thyrotropin in thyroid cancer patients. Clin Endocrinol (Oxf). 2006; 65:519-523.

16. Freudenberg LS, Jentzen W, Petrich T, et al. Lesion dose in differentiated thyroid carcinoma metastases after rhTSH or thyroid hormone withdrawal: ${ }^{124} \mathrm{I}$ PET/CT dosimetric comparisons. Eur J Nucl Med Mol Imaging. 2010;37:2267-2276.

17. Giovanella L, Duntas LH. Management of endocrine disease: the role of rhTSH in the management of differentiated thyroid cancer — pros and cons. Eur J Endocrinol. 2019;181:R133-R145.

18. Zanotti-Fregonara P, Hindie E. On the effectiveness of recombinant human TSH as a stimulating agent for ${ }^{131} \mathrm{I}$ treatment of metastatic differentiated thyroid cancer. Eur J Nucl Med Mol Imaging. 2010;37:2264-2266.

19. Plyku D, Hobbs RF, Huang K, et al. Recombinant human thyroid-stimulating hormone versus thyroid hormone withdrawal in ${ }^{124} \mathrm{I}$ PET/CT-based dosimetry for ${ }^{131} \mathrm{I}$ therapy of metastatic differentiated thyroid cancer. J Nucl Med. 2017;58:1146-1154.

20. Durante C, Haddy N, Baudin E, et al. Long-term outcome of 444 patients with distant metastases from papillary and follicular thyroid carcinoma: benefits and limits of radioiodine therapy. J Clin Endocrinol Metab. 2006;91:2892-2899.

21. Schmidt D, Linke R, Uder M, Kuwert T. Five months' follow-up of patients with and without iodine-positive lymph node metastases of thyroid carcinoma as disclosed by ${ }^{131} \mathrm{I}$-SPECT/CT at the first radioablation. Eur J Nucl Med Mol Imaging. 2010;37:699-705

22. Avram AM, Fig LM, Frey KA, Gross MD, Wong KK. Preablation 131-I scans with SPECT/CT in postoperative thyroid cancer patients: what is the impact on staging? J Clin Endocrinol Metab. 2013;98:1163-1171.

23. Avram AM, Esfandiari NH, Wong KK. Preablation 131-I scans with SPECT/CT contribute to thyroid cancer risk stratification and 131-I therapy planning. $J$ Clin Endocrinol Metab. 2015;100:1895-1902.

24. Avram AM, Rosculet N, Esfandiari NH, et al. Differentiated thyroid cancer outcomes after surgery and activity-adjusted ${ }^{131}$ I theragnostics. Clin Nucl Med. 2019; $44: 11-20$.
25. McDougall IR. $74 \mathrm{MBq}$ radioiodine ${ }^{131} \mathrm{I}$ does not prevent uptake of therapeutic doses of ${ }^{131}$ I (i.e. it does not cause stunning) in differentiated thyroid cancer. Nucl Med Commun. 1997;18:505-512.

26. Avram AM, Dewaraja YK. Thyroid cancer radiotheragnostics: the case for activity adjusted ${ }^{131} \mathrm{I}$ therapy. Clin Transl Imaging. 2018;6:335-346.

27. Jentzen W, Freudenberg L, Bockisch A. Quantitative imaging of ${ }^{124}$ I with PET/CT in pretherapy lesion dosimetry: effects impairing image quantification and their corrections. Q J Nucl Med Mol Imaging. 2011;55:21-43.

28. Luster M, Clarke SE, Dietlein M, et al. Guidelines for radioiodine therapy of differentiated thyroid cancer. Eur J Nucl Med Mol Imaging. 2008;35:1941-1959.

29. Sisson JC. Practical dosimetry of ${ }^{131} \mathrm{I}$ in patients with thyroid carcinoma. Cancer Biother Radiopharm. 2002;17:101-105.

30. Campennì A, Giovanella L, Pignata SA, et al. Undetectable or low $(<1 \mathrm{ng} / \mathrm{ml})$ postsurgical thyroglobulin values do not rule out metastases in early stage differentiated thyroid cancer patients. Oncotarget. 2018;9:17491-17500.

31. Bernier MO, Withrow DR, Berrington de Gonzalez A, et al. Trends in pediatric thyroid cancer incidence in the United States, 1998-2013. Cancer. 2019; 125:2497-2505.

32. Richman DM, Benson CB, Doubilet PM, et al. Assessment of American College of Radiology thyroid imaging reporting and data system (TI-RADS) for pediatric thyroid nodules. Radiology. 2020;294:415-420.

33. Francis GL, Waguespack SG, Bauer AJ, et al. Management guidelines for children with thyroid nodules and differentiated thyroid cancer. Thyroid. 2015;25: 716-759.

34. Zong Y, Li K, Dong K, Yao W, Liu G, Xiao X. The surgical choice for unilateral thyroid carcinoma in pediatrics: lobectomy or total thyroidectomy? J Pediatr Surg. 2018;53:2449-2453.

35. Parisi MT, Eslamy H, Mankoff D. Management of differentiated thyroid cancer in children: focus on the American Thyroid Association pediatric guidelines. Semin Nucl Med. 2016;46:147-164.

36. Machac J. Thyroid cancer in pediatrics. Endocrinol Metab Clin North Am. 2016; 45:359-404.

37. Sugino K, Nagahama M, Kitagawa W, et al. Papillary thyroid carcinoma in children and adolescents: long-term follow-up and clinical characteristics. World $J$ Surg. 2015;39:2259-2265.

38. Remiker AS, Chuang J, Corathers S, et al. Differentiated thyroid cancer in the pediatric/adolescent population: evolution of treatment. J Pediatr Hematol Oncol. 2019;41:532-536.

39. Cabanillas ME, McFadden DG, Durante C. Thyroid cancer. Lancet. 2016;388: 2783-2795.

40. Ain KB. Papillary thyroid carcinoma: etiology, assessment, and therapy. Endocrinol Metab Clin North Am. 1995;24:711-760.

41. Xing M, Haugen BR, Schlumberger M. Progress in molecular-based management of differentiated thyroid cancer. Lancet. 2013;381:1058-1069.

42. Robbins RJ, Wan Q, Grewal RK, et al. Real-time prognosis for metastatic thyroid carcinoma based on 2-[ $\left[{ }^{18} \mathrm{~F}\right]$ fluoro-2-deoxy-D-glucose-positron emission tomography scanning. J Clin Endocrinol Metab. 2006;91:498-505.

43. Giovanella L, van Nostrand D. Advanced differentiated thyroid cancer: when to stop radioiodine? Q J Nucl Med Mol Imaging. 2019;63:267-270.

44. Silberstein EB. The problem of the patient with thyroglobulin elevation but negative iodine scintigraphy: the TENIS syndrome. Semin Nucl Med. 2011;41: $113-120$.

45. Schleupner MC, Riemann B, Schafers M, Backhaus P, Vrachimis A. Impact of FDG-PET on therapy management and outcome of differentiated thyroid carcinoma patients with elevated thyroglobulin despite negative iodine scintigraphy. Nuklearmedizin. 2020;59:356-364.

46. Wang W, Larson SM, Fazzari M, et al. Prognostic value of $\left[{ }^{18} \mathrm{~F}\right]$ fluorodeoxyglucose positron emission tomographic scanning in patients with thyroid cancer. J Clin Endocrinol Metab. 2000;85:1107-1113.

47. Giovanella L, Scappaticcio L. Radioiodine therapy of advanced differentiated thyroid cancer: clinical considerations and multidisciplinary approach. $Q \mathrm{~J} \mathrm{Nucl} \mathrm{Med}$ Mol Imaging. 2019;63:229-234.

48. Porter A, Wong DJ. Perspectives on the treatment of advanced thyroid cancer: approved therapies, resistance mechanisms, and future directions. Front Oncol. 2021;10:592202

49. Sabra MM, Sherman EJ, Tuttle RM. Tumor volume doubling time of pulmonary metastases predicts overall survival and can guide the initiation of multikinase inhibitor therapy in patients with metastatic, follicular cell-derived thyroid carcinoma. Cancer. 2017;123:2955-2964.

50. Jaber T, Waguespack SG, Cabanillas ME, et al. Targeted therapy in advanced thyroid cancer to resensitize tumors to radioactive iodine. J Clin Endocrinol Metab. 2018;103:3698-3705. 